\title{
DETERMINAÇÃO in Loco DA DIFUSIVIDADE TÉRMICA NUM SOLO DA REGIÃO DE CAATINGA (PB) ${ }^{(1)}$
}

\author{
Alain Passerat de Silans ${ }^{(2)}$, Fernando Moreira da Silva ${ }^{(3)}$ \& Francisco \\ de Assis dos Reis Barbosa ${ }^{(4)}$
}

\begin{abstract}
RESUMO
Um estudo experimental detalhado sobre transferências de calor e água no complexo solo - vegetação - atmosfera, em uma região de caatinga no semi-árido paraibano, mostrou que o comportamento termodinâmico do solo exerce papel fundamental no processo de evaporação do solo e nos fluxos de calor sensível. Este trabalho objetivou mostrar como a difusividade térmica do solo in loco foi determinada. Três métodos diferentes, embasados sobre hipóteses bem distintas, foram utilizados, e os seus resultados foram comparados. O método harmônico não se mostrou adequado para o tipo de solo encontrado. Para a camada superficial do solo (0-5 cm), o método CLTM (Corrected Laplace Transform Method) mostrou-se bem adaptado. Em todos os casos, o método NHS (método de Nassar \& Horton), que considera variações verticais da difusividade térmica, apresentou uma dispersão elevada dos seus pontos, porém forneceu valores próximos dos valores estimados pelo método CLTM na camada superior e valores coerentes nas outras camadas. A umidade do solo, na profundidade média de $5 \mathrm{~cm}$, foi medida por uma sonda TDR devidamente calibrada. Assim, pôde se determinar a relação entre a difusividade térmica e a umidade volumétrica para o solo estudado. Os valores muito baixos de difusividade constituem um condicionante importante do clima local.
\end{abstract}

Termos de indexação: semi-árido, temperatura do solo, método harmônico, método de Laplace, método de Nassar e Horton.

\footnotetext{
(1) Estudo Experimental com Financiamento do CNPQ. Recebido para publicação em março de 2003 e aprovado em fevereiro de 2005.

(2) Professor do Centro de Tecnologia, Laboratório de Recursos hídricos e Engenharia Ambiental - LARHENA, Universidade Federal da Paraíba - UFPB. CEP 58059-900 João Pessoa (PB). E-mail: alain@lrh.ct.ufpb.br

(3) Professor do Departamento de Geografia, Universidade Federal do Rio Grande do Norte - UFRN. E-mail: fmoreira@npd.ufrn.br

(4) Estudante de Iniciação Científica, UFPB. Bolsista PIBIC.
} 


\title{
SUMMARY: In Loco DETERMINATION OF THE THERMAL DIFFUSIVITY IN A SOIL OF THE CAATINGA REGION, PARAIBA
}

\begin{abstract}
An in-depth experimental study into heat and water transference in the soil-vegetationatmosphere complex was carried out in a semi-arid region dominated by Caatinga (Xerophytic) vegetation in the state of Paraiba, Brazil. Results showed that the thermodynamic soil behaviour plays a fundamental role in soil evaporation and sensitive heat fluxes. In this paper we show how the thermal difusivity of the soil was calculated in situ by three different methods with different hypotheses. The harmonic method (HM) was not suitable for the studied soil. The Corrected Laplace Transform Method (CLTM) proved adequate only for the upper 0-5 cm soil layer. The points were greatly dispersed when using the NHS method, which considers vertical variability of the soil thermal diffusivity yields, although the results were similar to those of the CLTM method in the upper soil layer and coherent in the other layers. Soil moisture was measured with an adequately calibrated TDR probe at a depth of about $5 \mathrm{~cm}$. The thermal diffusivity could thus be related to the volumetric moisture of the soil under study. The very low values of thermal diffusivity represent an important component of the local climate.
\end{abstract}

Index terms: semi-arid region, soil temperature, harmonic method, Laplace method, Nassar and Horton method.

\section{INTRODUÇÃO}

A caatinga cobre quase todo o nordeste brasileiro, atingindo uma área de $800 \mathrm{mil} \mathrm{km}^{2}$, sendo interrompida por algumas áreas de floresta tropical úmida e cerrado. A irregularidade climática é sua característica principal, apresentando um prolongado período de seca, que se reflete na paisagem.

Apesar da aparência árida e pobre, a caatinga se revela como um ecossistema complexo, pela capacidade de adaptação de seus seres vivos à acentuada aridez do território. Além disso, apresenta solos relativamente férteis que, pela sua alta biodiversidade, potencializa a região com abundantes recursos genéticos.

A formação vegetal apresenta heterogeneidade quanto à sua aparência e composição. Em alguns trechos, exibe uma mata rala ou aberta, daí o nome caatinga, que, na linguagem indígena, significa mata branca; em outros, o solo aparece quase descoberto, mostrando arbustos isolados.

A região apresenta duas estações pouco definidas: uma quente e seca, no inverno, e, outra, quente com chuvas, no verão-outono. Durante o período da estiagem, a vegetação apresenta um aspecto seco, sem folhagens, e o solo pedregoso exibe raízes. É uma adaptação das plantas às condições climáticas. Dessa forma, as folhas finas que se desprendem das árvores fazem com que a planta diminua a transpiração, evitando a perda de água, e suas raízes, permanecendo na superfície do solo, por sua vez, absorvem mais rapidamente a água das chuvas.

A complexa interação entre o solo, a vegetação e a atmosfera precisa ser bem entendida para melhorar as previsões meteorológicas e as respostas dos modelos hidrológicos e ecológicos. Experimentos foram propostos nestas duas últimas décadas, patrocinados pelo IGBP (International GeosphereBiosphere Programme), para analisar os processos em questão e os métodos para quantificá-los. Destes experimentos, podem ser destacados: ABRACOS, na floresta amazônica Brasileira; FIFE, em uma região temperada dos Estados Unidos, HAPEX-Mobilhy, em uma região temperada da França; HAPEX-Sahel, em uma região semi-árida da África Ocidental; BOREAL, na região pré-ártica, e LBA, na região amazônica.

Com objetivos similares para a região semi-árida do Nordeste Brasileiro, instalou-se, no município de São João do Cariri na Paraíba, um experimento por meio do qual, em um local representativo da região, foram medidos os balanços de radiação, de energia e hídrico. O lay-out experimental foi descrito em Silva et al. (2000). Silans et al. (2003) mostraram que, neste experimento, os fluxos de calor sensível e de vapor de água entre o solo e a copa da vegetação se processam por convecção livre. As diferenças de temperatura e pressão de vapor entre a superfície do solo e a copa da vegetação, neste caso, condicionam as taxas de transferência de calor e de evaporação a partir do solo (Massman, 1992; Jacobs \& Verhoef, 1997).

Neste contexto, percebe-se que o comportamento termodinâmico da vegetação e do solo apresenta grande importância. A difusividade térmica do solo reflete sua capacidade em transportar o calor para dentro. A uma difusividade baixa, por exemplo, podem-se associar uma temperatura máxima alta na superfície e pequena profundidade de penetração 
do calor. A difusividade térmica do solo varia com o tempo e espaço. Ela depende, dentre outros fatores, da constituição, granulometria, densidade e estrutura do solo, propriedades estas que, na escala de tempo do experimento ou dos processos hidrológicos, não variam com o tempo, mas podem variar com a profundidade, e da umidade volumétrica do solo que varia rapidamente com o tempo, sobretudo nas camadas superficiais. Diversos métodos foram publicados na literatura para determinar in loco a difusividade térmica do solo.

Neste trabalho, a difusividade térmica do solo foi determinada para o solo Bruno não-Cálcico do experimento já referido, utilizando três métodos com embasamentos teóricos diferentes. Discutiu-se a adequação dos métodos estudados ao tipo de solo e às condições muito específicas de semi-aridez da região.

\section{MATERIAL E MÉTODOS}

\section{Aspectos teóricos}

A equação unidimensional vertical da condução de calor no solo é dada por:

$$
\mathrm{C} \frac{\partial \mathrm{T}}{\partial \mathrm{t}}=-\frac{\partial}{\partial \mathrm{z}}\left(-\lambda \frac{\partial \mathrm{T}}{\partial \mathrm{z}}\right)
$$

em que $\mathrm{C}$ é a capacidade calorífica do solo $\left(\mathrm{Jm}^{-3} \mathrm{~K}^{-1}\right)$ e $\lambda$, a condutividade térmica aparente $\left(\mathrm{Wm}^{-1} \mathrm{~K}^{-1}\right)$. Admitindo que a capacidade calorífica não varia com a profundidade $\mathrm{z}$, a equação (1) se torna:

$$
\frac{\partial \mathrm{T}}{\partial \mathrm{t}}=\frac{\partial}{\partial \mathrm{z}}\left(\mathrm{K} \frac{\partial \mathrm{T}}{\partial \mathrm{z}}\right)
$$

em que $\mathrm{K}$ é a difusividade térmica aparente do solo $\left(\mathrm{m}^{2} \mathrm{~s}^{-1}\right)$. O termo aparente foi introduzido por De Vries (1963). Esse autor analisou em detalhe o processo de transferência de calor no solo e mostrou que, além de um processo essencialmente condutivo pelos contatos físicos entre as fases sólida, líquida e gasosa, nos espaços intraporais, existe transferência de calor por convecção associada a processos de evaporação-condensação. Para maiores detalhes, consultar Passerat de Silans (1986). A condutividade térmica e a difusividade térmica medidas in loco representam esses processos, motivo pelo qual elas são chamadas de aparentes.

Segundo a literatura (De Vries, 1963; Horton et al., 1983; Passerat de Silans et al., 1996), vários métodos têm sido utilizados com o objetivo de quantificar a difusividade térmica e, para tanto, utilizam dados de temperatura do solo em diferentes profundidades. Neste trabalho, os métodos harmônico (HM), da Transformada de Laplace corrigido (CLTM) e de Nassar e Horton (NHS) são utilizados e descritos a seguir.

\section{O método harmônico (HM)}

Trata-se do método mais utilizado, por ser fácil de programá-lo no computador e por sua adaptação às regiões temperadas. A temperatura medida na superfície pode, geralmente, ser descrita por uma decomposição em série de Fourier:

$$
\mathrm{T}(0, \mathrm{t})=\overline{\mathrm{T}}+\sum_{\mathrm{i}=1}^{\mathrm{N}} \mathrm{A}_{\mathrm{i}} \operatorname{sen}\left(\mathrm{i} \omega \mathrm{t}+\phi_{\mathrm{i}}\right)
$$

em que $\overline{\mathrm{T}}$ representa a temperatura média no período $\mathrm{P}$ (aqui $\mathrm{P}=24 \mathrm{~h}$ ), $\mathrm{A}_{\mathrm{i}}$ e $\phi_{\mathrm{i}}$ representam, respectivamente, a amplitude e a fase da harmônica i. O termo $\omega$ representa a freqüência fundamental.

A solução analítica da equação de condução de calor (equação 1), considerando $\lambda$ e C constantes, é dada por:

$$
\mathrm{T}(\mathrm{z}, \mathrm{t})=\overline{\mathrm{T}}+\sum_{\mathrm{i}=1}^{\mathrm{N}} \mathrm{A}_{\mathrm{i}} \exp \left(\frac{-\mathrm{z}}{\mathrm{d}_{\mathrm{i}}}\right) \operatorname{sen}\left(\mathrm{i} \omega \mathrm{t}+\phi_{\mathrm{i}}-\frac{\mathrm{z}}{\mathrm{d}_{\mathrm{i}}}\right)
$$

em que $\exp \left(\frac{-\mathrm{z}}{\mathrm{d}_{\mathrm{i}}}\right)$ e'( $\left(\frac{-\mathrm{z}}{\mathrm{d}_{\mathrm{i}}}\right)$ representam, respectivamente, o amortecimento e a defasagem para cada harmônica. Nesta equação, $\mathrm{d}_{\mathrm{i}}=\sqrt{2 \mathrm{~K} / \mathrm{i} \omega}$ representa, segundo van Wijk (1963), a profundidade de penetração da onda de calor durante o período $\mathrm{P} / \mathrm{i}$.

A difusividade térmica $(K)$ é identificada a cada dia por meio de uma técnica de ajuste, procurando minimizar uma função critério. A função critério escolhida geralmente corresponde ao erro quadrático médio entre os valores observados em determinada profundidade z e o valor calculado pela equação (4).

$\mathrm{Na}$ utilização deste método, considera-se a hipótese de que a difusividade térmica é, ao longo de um período, constante no tempo e na vertical, até uma profundidade teoricamente infinita. Observa-se, também, que a solução analítica da equação da condução do calor não necessita das condições iniciais sobre o perfil vertical de temperatura, uma vez que a hipótese de estabilidade da periodicidade para todas as harmônicas é considerada, ou seja, a onda da temperatura na superfície conserva a periodicidade de suas diversas harmônicas por longo tempo, até mesmo anterior ao período examinado. Assim, o perfil inicial não apresenta mais influência sobre os perfis verticais de temperatura nos períodos estudados. Teoricamente, este fato admite ser a temperatura média diária a mesma em todas as profundidades.

O método da transformada de Laplace corrigido (CLTM)

Considerando a linearidade da eq. (2), Passerat de Silans (1988) propõe uma solução analítica desta equação pela superposição da solução analítica de dois problemas:

1 - com uma condição nula para o limite superior do domínio e um perfil inicial de temperatura, sendo função de z: 


$$
\begin{aligned}
& \mathrm{T}(0, \mathrm{t})=0 \\
& \mathrm{~T}(\mathrm{z}, 0)=\mathrm{F}(\mathrm{z})
\end{aligned}
$$

2 - com uma temperatura na superfície função do tempo t, condição no limite superior do domínio e um perfil inicial de temperatura nulo:

$$
\begin{aligned}
& \mathrm{T}(0, \mathrm{t})=\phi(\mathrm{t}) \\
& \mathrm{T}(\mathrm{z}, 0)=0
\end{aligned}
$$

A solução do primeiro problema é obtida de Carslaw \& Jaeger (1959) por meio da teoria das imagens:

$$
\mathrm{T}_{1}(\mathrm{z}, \mathrm{t})=\frac{1}{\sqrt{\pi \mathrm{Kt}}} \int_{0}^{\infty} \mathrm{F}\left(\mathrm{z}^{\prime}\right)\left\{\exp \left(-\frac{\left(\mathrm{z}-\mathrm{z}^{\prime}\right)^{2}}{4 \mathrm{Kt}}\right)-\exp \left(-\frac{\left(\mathrm{z}+\mathrm{z}^{\prime}\right)^{2}}{4 \mathrm{Kt}}\right)\right\} d z^{\prime}(9)
$$

enquanto o segundo problema é resolvido analiticamente, utilizando a transformada de Laplace (Passerat de Silans, 1988):

$$
\mathrm{T}_{2}(\mathrm{z}, \mathrm{t})=\frac{\mathrm{z}}{2 \sqrt{\pi \mathrm{K}}} \int_{0}^{\mathrm{t}} \Phi(\tau) \frac{\exp \left(-\frac{\mathrm{z}^{2}}{4 \mathrm{~K}(\mathrm{t}-\tau)}\right)}{(\mathrm{t}-\tau)^{3 / 2}} \mathrm{~d} \tau
$$

A partir de certa profundidade $\mathrm{z}_{0}$, a qual depende da difusividade térmica, a temperatura $\mathrm{T}_{\mathrm{z} 0}$ praticamente não varia no tempo, na escala de um experimento como este realizado em São João do Cariri. É razoável admitir que a temperatura, a partir desta profundidade, seja próxima da temperatura média da superfície. Assim, o conjunto dos dois problemas aplica-se à difusão do calor no solo, se, na equação (9), substituir-se $\mathrm{F}(\mathrm{z})$ por $\mathrm{f}(\mathrm{z})=$ $\mathrm{F}(\mathrm{z})-\mathrm{T}_{\mathrm{z} 0} \mathrm{e}$, na equação 10 , admitir-se que $\Phi(\tau)=$ $\mathrm{T}(0, \mathrm{t})-\mathrm{T}_{\mathrm{z} 0}$.

Com estas considerações, T(z,t) no solo será dada pela expressão:

$$
\mathrm{T}(\mathrm{z}, \mathrm{t})=\mathrm{T}_{\mathrm{z} 0}+\mathrm{T}_{1}(\mathrm{z}, \mathrm{t})+\mathrm{T}_{2}(\mathrm{z}, \mathrm{t})
$$

Utiliza-se então a mesma técnica para determinar a difusividade térmica apresentada para o método harmônico. A vantagem desse método em relação ao método harmônico é que a minimização do erro quadrático médio pode ser feito sobre um tempo bem inferior ao período do fenômeno, tendo, assim, maior possibilidade de respeitar a hipótese fundamental da constância da condutividade térmica e da capacidade térmica.

Passerat de Silans (1988), efetuando um estudo de sensibilidade detalhado, mostrou que, ao escolherse um perfil de temperatura logo que a temperatura da superfície começa pela manhã a subir significativamente, encontram-se condições adequadas à aplicação da Transformada de Laplace (problema 2), ao mesmo tempo que, geralmente, a umidade do solo sofre uma redistribuição durante a noite no sentido de uma uniformização do seu perfil vertical.
Além disto, o método é aplicável quando a periodicidade não se mostra estacionária, isto é, quando modificações bastante bruscas ocorrem nas condições ambientais do solo (modificações climáticas bruscas ou irrigação, por exemplo). Vale salientar que o método foi aplicado satisfatoriamente, durante a secagem de um solo descoberto, numa região mediterrânea (Passerat de Silans et al., 1989), assim como na determinação da difusividade térmica de um solo no experimento HAPEX-Sahel, realizado em uma região semi-árida da África (Passerat de Silans et al., 1996).

\section{O método de Nassar \& Horton (NHS)}

Os dois métodos apresentados anteriormente consideram o solo homogêneo quanto a suas propriedades térmicas. Entretanto, fortes gradientes de umidade existem durante o dia na zona não saturada do solo, principalmente na proximidade de sua superfície. Estratificações verticais da densidade global, $d_{g}$ são freqüentemente encontradas nos solos pouco profundos das regiões semi-áridas. Nestes casos, o solo não é homogêneo verticalmente.

Nassar \& Horton (1989) aplicaram a teoria de Lettau para a difusividade térmica de um solo nãohomogêneo (Lettau, 1954). Considerando apenas uma harmônica para descrever a temperatura na superfície do solo, assim como o fluxo de calor, G, Lettau verificou que, em ambos os casos, as amplitudes e fases em diversas profundidades dependem da profundidade $\mathrm{z}$ :

$$
\begin{aligned}
& \mathrm{T}(\mathrm{z}, \mathrm{t})=\mathrm{T}_{\mathrm{m}}+\mathrm{A}(\mathrm{z}) \operatorname{sen}(\omega \mathrm{t}+\phi(\mathrm{z})) \\
& \mathrm{G}(\mathrm{z}, \mathrm{t})=\mathrm{G}_{\mathrm{m}}+\mathrm{B}(\mathrm{z}) \operatorname{sen}(\omega \mathrm{t}+\beta(\mathrm{z}))
\end{aligned}
$$

em que $\mathrm{T}_{\mathrm{m}}$ e $\mathrm{G}_{\mathrm{m}}$ representam a temperatura média e o fluxo de calor médio, respectivamente, durante o período do fenômeno. Isto evidencia que tanto a temperatura média como o fluxo de calor médio não variam com a profundidade. O fluxo de calor G é relacionado com a temperatura $\mathrm{T}$, pelas duas expressões:

$$
\begin{aligned}
& G(z, t)=-\left.\lambda \frac{\partial T}{\partial z}\right|_{Z} \\
& \frac{\partial G}{\partial z}=-C \frac{\partial T}{\partial t}
\end{aligned}
$$

Nassar \& Horton (1989), aplicando a teoria de Lettau (1954), mostraram que a difusividade térmica, K, podia ser calculada, considerando os perfis verticais das amplitudes $\mathrm{A}(\mathrm{z})$ e $\mathrm{B}(\mathrm{z})$ e das fases $\phi(z)$ e $\beta(z)$. Segundo esses autores, o ajuste destes parâmetros calculados nas diversas profundidades em que a temperatura $\mathrm{T}(\mathrm{z}, \mathrm{t})$ é medida, por uma decomposição em série de Fourier a uma harmônica, deve ser feito por uma função spline cúbico para se obter maior precisão sobre os resultados, já que a expressão da difusividade térmica envolve as derivadas desses parâmetros em relação à profundidade $\mathrm{z}$ : 


$$
\mathrm{K}(\mathrm{z})=\frac{\lambda(\mathrm{z})}{\mathrm{C}}=\frac{\omega \operatorname{sen}(\varepsilon(\mathrm{z})) \operatorname{sen}(\delta(\mathrm{z}))}{\frac{\partial \phi(\mathrm{z})}{\partial \mathrm{z}} \frac{\partial \beta(\mathrm{z})}{\partial \mathrm{z}}}
$$

em que $\varepsilon(z)$ é dado pela expressão:

$$
\begin{gathered}
\varepsilon(\mathrm{z})=\beta(\mathrm{z})-\phi(\mathrm{z})-\pi, \mathrm{e} \\
\delta(\mathrm{z})=\phi(\mathrm{z})-\beta(\mathrm{z})-\frac{\pi}{2}
\end{gathered}
$$

A dedução dessas equações, assim como maiores detalhes sobre o método, encontram-se em Passerat de Silans et al. (1996). Nesta dedução, admite-se que somente a condutividade térmica varia com a profundidade. A capacidade calorífica permanece constante.

\section{Estudo experimental}

O experimento foi instalado na bacia escola da Universidade Federal da Paraíba na Cidade de São João do Cariri, no estado da Paraíba, geograficamente situada na região dos Cariris Velhos. Trata-se de uma região com solos predominantemente Bruno não-cálcicos e a vegetação do tipo caatinga é predominantemente composta por: pastagem (capim mimoso); plantas arbóreas (marmeleiro, mucunfo, pinhão branco); plantas lenheiras (angico, catingueira) e cactáceas (facheiro, palmatória, palma doce e cardeiro). Trata-se de uma região classificada como semi-árida ( índice de aridez de 0,21 segundo Souza, 1999), com chuvas pouco abundantes e concentradas nos meses de fevereiro a abril. O solo tem aparência pedregosa e a vegetação é esparsa, alternando-se ora com superfície desnudada, ora com solo coberto por vegetação.

O experimento foi projetado para realizar, por períodos de 20 min o balanço radiativo, o balanço de energia e o balanço hídrico. Os dados foram coletados em um sistema de aquisição de dados alimentado em contínuo por uma bateria de $12 \mathrm{v}$ e $55 \mathrm{AH}$ acoplada a um painel solar com potência de $20 \mathrm{~W}$.

O sistema foi também programado para controlar todo o experimento. Uma torre com $8 \mathrm{~m}$ de altura foi erguida no meio da vegetação para coletar informações acima da vegetação, dentro da camada limite e dentro da copa da vegetação. No solo, medições de temperatura e do fluxo de calor foram efetuadas. O sensor de temperatura utilizado foi constituído de um termistor, operando numa faixa de -3 a $90{ }^{\circ} \mathrm{C}$, com precisão de $0,001{ }^{\circ} \mathrm{C}$, sendo instalados um na superfície e os outros nas profundidades $2,5,15$ e $50 \mathrm{~cm}$. Uma bateria de sensores foi aterrada debaixo de uma superfície coberta por vegetação e a outra debaixo de uma superfície desnudada. Os fluxos de calor foram medidos por um fluxômetro de tipo pastilha, localizado a $5 \mathrm{~cm}$ de profundidade.

O solo, pouco profundo, é estratificado. Medições da densidade do solo, $\mathrm{d}_{\mathrm{g}}$, foram feitas em todas as profundidades entre 0 e $40 \mathrm{~cm}$, considerando
15 amostras indeformadas por profundidade explorada, as quais mostraram que o solo podia ser dividido em duas camadas (Figura 1). A análise granulométrica revelou um solo franco-arenoso em todas as profundidades.

Um pouco mais de um ano de dados são disponíveis atualmente. No dia 6 de novembro de 2001, a área descoberta onde se encontram os sensores de temperatura e de fluxo de calor foi saturada em água. Após um intervalo de $24 \mathrm{~h}$, medições da umidade (\% em massa) foram feitas, retirando amostras com trado em torno dos sensores, nas profundidades médias de 2,5, 5, 10, 20, 30 e $40 \mathrm{~cm}$. Com esses valores, a sonda TDR centrada na profundidade de $5 \mathrm{~cm}$ foi calibrada.

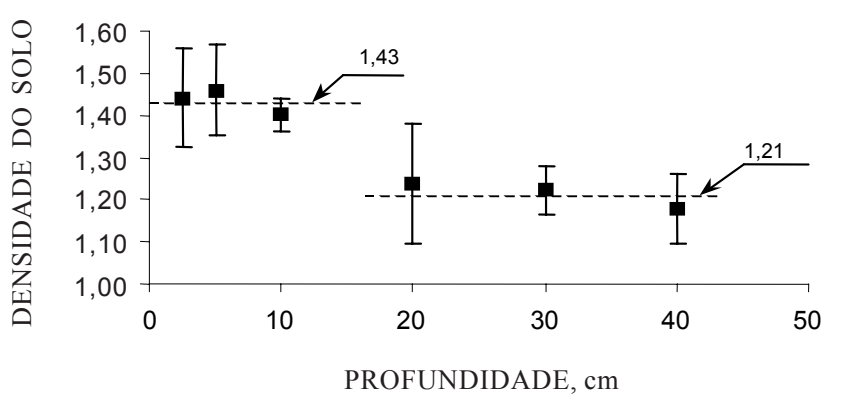

Figura 1. Perfil da densidade do solo. O perfil evidencia duas camadas.

\section{RESULTADOS E DISCUSSÃO}

Uma análise inicial foi efetuada sobre a distribuição das temperaturas no solo no período de 9 a 12/11/2001. Nesse período, o solo estava úmido, apesar da ausência de chuvas por várias semanas. A umidade se deu por meio do processo de irrigação. Em 6 de novembro de 2001, a parcela não recoberta por vegetação foi molhada até à saturação. A partir do dia 7/11/2001, acompanhou-se o processo de secagem deste solo. A figura 2 representa a evolução das temperaturas do solo na superfície e nas profundidades de 2,5 e $15 \mathrm{~cm}$.

Observou-se que a temperatura na profundidade de $15 \mathrm{~cm}$ mostrou-se quase constante, evidenciando que a onda de temperatura na superfície penetrava vagarosamente no solo. Da mesma forma, as temperaturas nas profundidades de 2 e $5 \mathrm{~cm}$ sofriam, em relação à temperatura na superfície, um forte amortecimento e uma defasagem muito significativa. Estes fatos revelam uma difusividade térmica do solo muito baixa.

Para aplicar os três métodos considerados na estimativa da difusividade térmica in loco, os sinais de temperaturas medidos nas diversas profundidades 
foram descritos por séries de Fourier. No caso dos métodos HM e CLTM, uma decomposição em séries de Fourier com seis harmônicas mostrou-se adequada (Figura 3). No caso do método NHS, utilizou-se uma decomposição a uma harmônica. Observou-se também o ajustamento aos dados experimentais da decomposição em séries de Fourier com uma harmônica em comparação com a decomposição com seis harmônicas (Figura 3).

Com os três métodos apresentados, calculou-se a difusividade térmica do solo $\mathrm{K}$ em vários intervalos de tempo distribuídos ao longo do experimento. Os intervalos de tempo foram escolhidos para representar o estudo sobre secagem do solo nos meses de novembro e dezembro 2001, após este ter sido saturado artificialmente, além de um período seco e outro imediatamente após um evento chuvoso em cada uma das estações anuais: a estação chuvosa, de janeiro a maio, e a estação seca, de junho a dezembro. Em cada um destes intervalos de tempo, a difusividade térmica e o erro quadrático médio (EQM) foram calculados com os métodos HM e CLTM, respectivamente. No caso deste último, para descrever o perfil inicial, considerou-se que a temperatura na profundidade de $20 \mathrm{~cm}$ não variou, apresentando um valor igual à média das temperaturas a $15 \mathrm{~cm}$. Com o método NHS, não se calcula um erro quadrático médio e a difusividade térmica é calculada em cada profundidade e não em uma camada de solo.

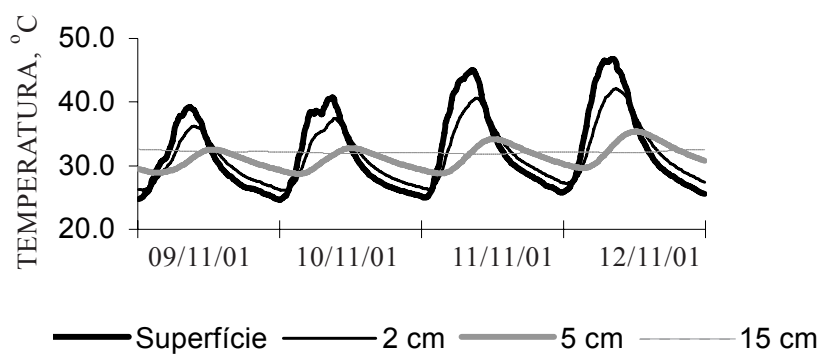

Figura 2. Evolução das temperaturas do solo na superfície e nas profundidades de 2,5 e $15 \mathrm{~cm}$.

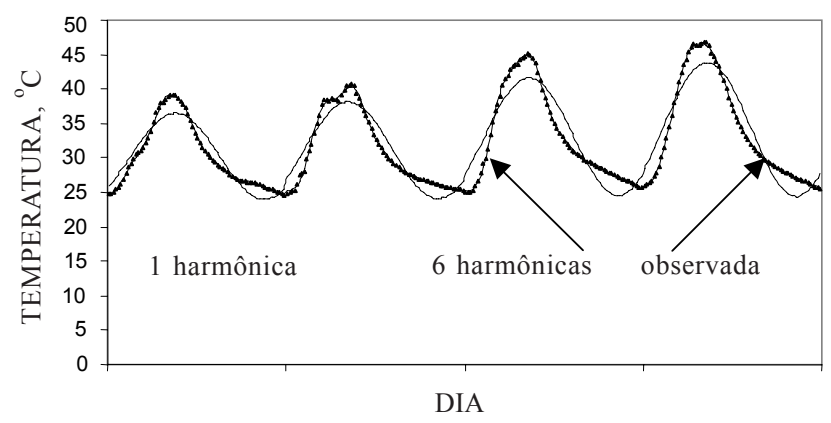

Figura 3. Ajustamento da temperatura de superfície por séries de Fourier a uma e seis harmônicas.
Assim, foram gerados perfis verticais de difusividade térmica, com 50 pontos, e estimaramse as difusividades térmicas nas camadas respectivas pela média aritmética dos valores de difusividade situados na camada considerada. Nas estimativas da difusividade térmica, o dia (período de cálculo) iniciou-se às 6:00 h da manhã. Para as camadas de solo de $0-5$; 0-15 e 5-15 cm, foi traçado o gráfico da difusividade térmica versus a umidade volumétrica do solo na profundidade média de $5 \mathrm{~cm}$ (Figuras 4 a 6). A umidade volumétrica foi medida a cada $20 \mathrm{~min}$ por uma sonda TDR, cujos dois eletrodos foram implantados horizontalmente nas profundidades de 2,5 e $7,5 \mathrm{~cm}$. A umidade média em torno de $5 \mathrm{~cm}$ às 11:00 h foi escolhida para compor os gráficos das figuras 4 a 6 . Em cada gráfico, os resultados dos três métodos são apresentados (Quadro 1):

Valores médios e o desvio-padrão do EQM foram calculados para os métodos HM e CLTM em cada camada considerada.

Observou-se (Quadro 1) que o valor do Erro Quadrático Médio (EQM) foi sempre bem menor

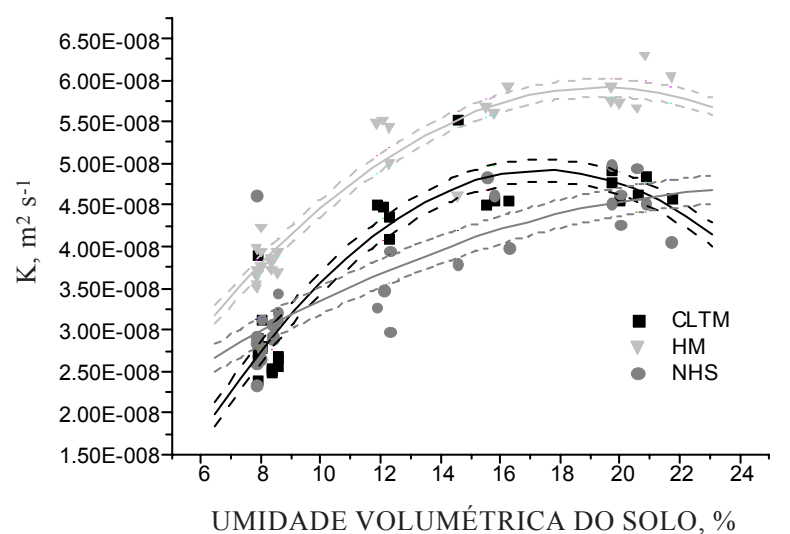

Figura 4. Difusividade térmica na camada de 0-5 cm, segundo os três métodos utilizados (CLTM, HM e NHS).

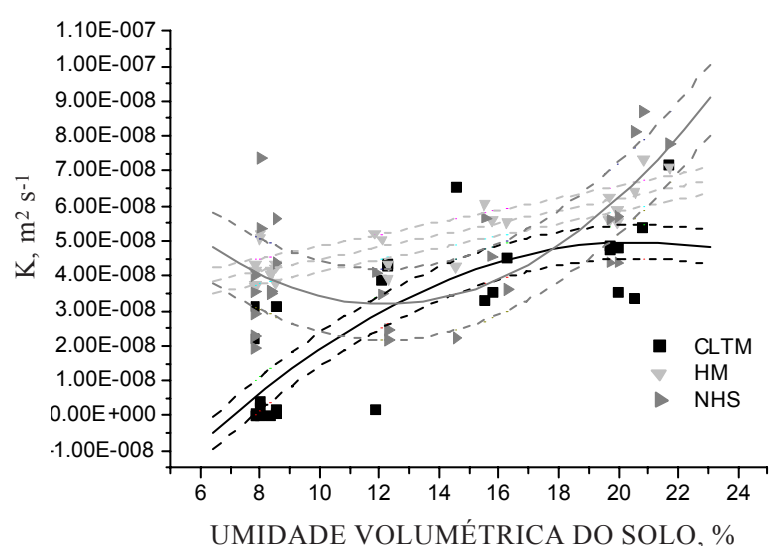

Figura 5. Difusividade térmica na camada de 0-15 cm, segundo os três métodos utilizados (CLTM, HM e NHS). 
Quadro 1. Média e desvio-padrão do erro quadrático médio

\begin{tabular}{|c|c|c|c|c|c|c|}
\hline \multirow{3}{*}{ EQM } & \multicolumn{6}{|c|}{ Camada do solo } \\
\hline & \multicolumn{2}{|c|}{$0-5 \mathrm{~cm}$} & \multicolumn{2}{|c|}{$0-15 \mathrm{~cm}$} & \multicolumn{2}{|c|}{$5-15 \mathrm{~cm}$} \\
\hline & HM & CLTM & HМ & CLTM & HМ & CLTM \\
\hline Média & 0,187 & 0,078 & 0,23 & 0,024 & 0,077 & 0,038 \\
\hline Desvio-padrão & 0,100 & 0,038 & 0,138 & 0,044 & 0,053 & 0,044 \\
\hline
\end{tabular}

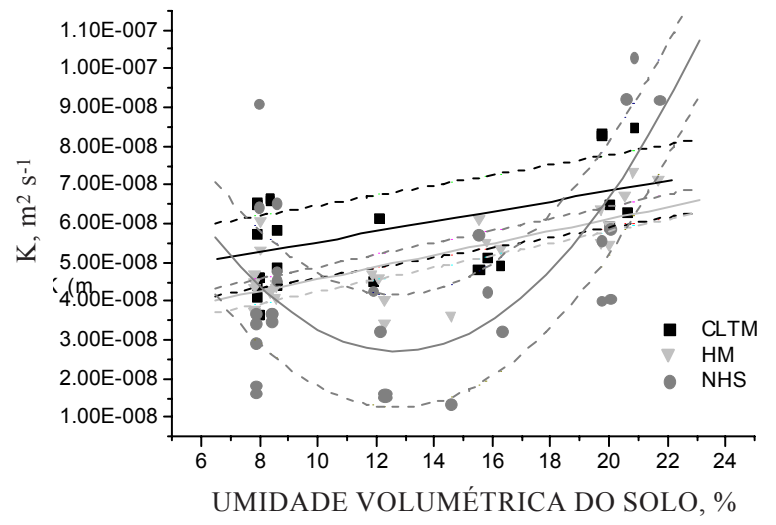

Figura 6. Difusividade térmica na camada de 5-15 cm, segundo os três métodos utilizados (CLTM, HM e NHS).

para o método CLTM do que para o método HM, indicando um ajuste da solução analítica aos valores observados sempre melhor com o método baseado sobre a transformada de Laplace. No entanto, a dispersão em torno da média foi para este método, na camada de $0-15 \mathrm{~cm}$, bem superior ao valor da média.

Nos gráficos das figuras 4 a 6 , uma função polinomial foi ajustada aos dados experimentais, bem como foi traçado o intervalo de confiança (95\%) com um nível de significância de $5 \%$ em torno da curva ajustada.

Na camada de $0-5 \mathrm{~cm}$, a difusividade térmica em relação à umidade volumétrica do solo mostrou-se semelhante para os três métodos considerados, e os valores calculados foram da mesma ordem de grandeza, confirmando o valor muito baixo da difusividade térmica, como esperado. Os valores de difusividade térmica calculados pelo método harmônico (HM) foram estatisticamente sempre superiores aos valores calculados pelos métodos CLTM e NHS, como se pode observar dos intervalos de confiança traçados. O Erro Quadrático Médio, EQM mostrou-se maior no método harmônico, HM, do que no método CLTM em qualquer estação considerada, chuvosa ou estiagem, e período considerado, seco ou úmido, em fase de secagem. Os valores apresentados pelos métodos CLTM e NHS foram bastante próximos, principalmente quando o solo revelou-se bastante seco ou bastante úmido. Para um solo com umidade média na camada de 0 $5 \mathrm{~cm}$, gradientes importantes de umidade ocorreram nesta camada, devendo o método NHS ser mais apropriado por considerar estratificação vertical da difusividade térmica.

$\mathrm{Na}$ camada de $0-15 \mathrm{~cm}$, o método CLTM apresentou valores de EQM muito baixos. No entanto, uma análise detalhada mostrou que, apesar de o erro quadrático médio ter sido de fato minimizado, a máxima e a mínima da solução analítica não corresponderam aos valores observados, tampouco a periodicidade do fenômeno respeitada. Admitindo um valor médio de $5.10^{-8} \mathrm{~m}^{2} \mathrm{~s}^{-1}$, a profundidade de penetração da onda de temperatura em $24 \mathrm{~h}$ seria da ordem de $5 \mathrm{~cm}$. O método CLTM é um método a resposta impulsional, não podendo ser então aplicável nesta camada. Os valores obtidos pelos métodos HM e NHS mostram-se bastante próximos, principalmente quando o solo, ora é seco, ora é bem úmido, apesar de os valores apresentados pelo método NHS serem muito dispersos nestas condições. Para uma umidade média na camada de 0-15 cm, gradientes importantes de umidade devem ocorrer e o método NHS deve ser mais apropriado por considerar estratificação vertical da difusividade térmica. Considerações idênticas devem ser feitas para a camada de 5 a $15 \mathrm{~cm}$.

Diante desses resultados, percebe-se que o método CLTM forneceu resultados mais confiantes na camada de $0-5 \mathrm{~cm}$. Nas outras camadas, os resultados deste método devem ser descartados. O método HM sempre apresentou valores elevados de EQM, mostrando ser pouco adequado para determinar a difusividade térmica neste solo. O método NHS apresentou na camada de $0-5 \mathrm{~cm}$ valores próximos aos do método CLTM, porém com maior dispersão. Nas outras camadas, este método apresentou valores confiáveis apenas quando a camada do solo revelou uma umidade média.

Utilizando os valores calculados pelo método CLTM na camada superior do solo, eliminando, neste caso, os pontos com valor de EQM superiores a $0,1{ }^{\circ} \mathrm{C}$, obteve-se o seguinte ajuste matemático para a difusividade térmica do solo Bruno não-Cálcico da região do Cariri Paraibano: 
$\mathrm{K}=-1,1910^{-13} \theta^{3}-2,6610^{-11} \theta^{2}+2,2710^{-9} \theta+1,3610^{-8}$ $\left(\mathrm{R}^{2}=0,82\right)$

em que $\mathrm{K}$ é a difusividade térmica em $\mathrm{m}^{2} \mathrm{~s}^{-1}$ e $\theta$ é a umidade volumétrica em percentagem.

Esta equação aplica-se ao primeiro estrato de solo: $0-17,5 \mathrm{~cm}$, já que ele se apresentou bastante uniforme, tanto considerando a densidade do solo como as curvas granulométricas.

\section{CONCLUSÕES}

1. Constatou-se, no solo analisado da região de caatinga no semi -árido do Cariri, a existência de gradientes verticais de temperatura muito fortes na superfície e uma pequena penetração da onda de temperatura. A defasagem entre as ondas de temperatura observadas na superfície e na profundidade de $5 \mathrm{~cm}$ foi muito grande, caracterizando um solo com muito baixa difusividade térmica. Este resultado é de fundamental importância para a compreensão do clima na região, do processo de evaporação do solo e do balanço hídrico. Utilizaramse os métodos Harmônico (HM), Laplace corrigido (CLTM) e Nassar e Horton's (NHS) para calcular a difusividade térmica in loco.

2. O método harmônico, no solo estudado, não forneceu respostas satisfatórias.

3. O método CLTM mostrou-se eficiente na camada de $0-5 \mathrm{~cm}$, mas inadequado nas demais camadas em virtude do baixo valor de difusividade térmica.

4. Já o método de Nassar e Horton, desenvolvido considerando possibilidade de variações verticais da difusividade térmica do solo, mostrou-se adequado em todas as camadas, quando a umidade média da camada de solo apresentou valores intermediários, e apresentou resultados similares aos do método CLTM na camada superficial, apesar de mais dispersos. Uma equação matemática traduzindo a dependência da difusividade térmica com a umidade volumétrica do solo foi determinada.

\section{AGRADECIMENTOS}

Os autores deste trabalho agradecem ao CNPq, pelo financiamento do estudo experimental (auxílio integrado proc. 479006/01-6). Agradecem também ao Dr.Antônio Ocimar Manzi, do INPE, pela visita ao experimento e discussões, assim como ao Dr. Nelson Dias do SIMEPAR, pelas várias correspondências mantidas. Agradecem também aos revisores anônimos deste trabalho, pelas sugestões e correções.

\section{LITERATURA CITADA}

CARSLAW, H.S. \& JAEGER, J.C. Conduction heat in solids. 2.ed. Oxford, Oxford Science Publications, 1959. 510p.

DE VRIES, D.A. Thermal properties of soil. In: WIJK, W.R. van, ed. Physics of plant environment. Amsterdam, North Holland, 1963. p.210-235.

JACOBS, A.F.G. \& VERHOEF, A. Soil evaporation from sparse natural vegetation estimated fro Sherwood Numbers. J. Hydrol., 188-189:443-452, 1997.

HORTON, R.; WIERENGA, P.J. \& NIELSEN, D.R. Evaluation of methods for determining apparent thermal diffusivity of soil near the surface. Soil Sci. Soc. Am. J., 47:23-32, 1983.

LETTAU, H.H. Improved models of thermal diffusion in the soil. Trans.Am. Geophys. Union, 35:121-132, 1954.

MASSMAN, W.J. A surface energy balance method for partitioning evapotranspiration data into plant and soil components for a surface with partial canopy cover. Water Res. Res., 28:1723-1732, 1992.

NASSAR, I.N. \& HORTON, R. Determination of the apparent difusity of a nomuniform soil. Soil Sci., 147:238-244, 1989.

PASSERAT DE SILANS, A.M.B. Transferts de masse et de chaleur dans un sol stratifé soumis à une excitation atmosphérique naturelle: Comparaison modéle experience. Grenoble, INPG, 1986. 205p. (Tese de Doutorado)

PASSERAT DE SILANS, A.M.B. Determinação das propriedades termodinâmicas de um solo in loco. Brasília, CNPq, 1988. 18p. (Report 404015/86-7)

PASSERAT DE SILANS, A.M.B.; BRUCKLER, L.; THONY, J.L. \& VAUCLIN, M. Numerical modeling of coupled heat and water flows during drying in a stratified bare soil. Comparison with field observations. J. Hydrol., 105:109-138, 1989.

PASSERAT DE SILANS, A.M.B.; MONTENY, B.A. \& LHOMME, J.P. Apparent soil thermal diffusivity: hapexsahel experiment. Agric. Forest Met., 81:201-216, 1996.

PASSERAT DE SILANS, A.M.B. \& SILVA, F.M. Bilans energétique et hydrique en une région semi-aride dominée par la Caatinga. In: CONFÉRENCE INTERNATIONALE: Hydrologie des régions méditerranéennes et semi-arides, 2003. Montepellier. Proceedings, Montepellier, 2003. CD ROM

SILVA, F.M.; PASSERAT DE SILANS, A.M.B.; PAZ, A.R.; GOMES, R.S. \& SOUZA, E.E. Estudo experimental da interação solo vegetação atmosfera na região do Cariri. In: SIMPÓSIO DE RECURSOS HÍDRICOS DO NORDESTE, 5., Natal, 2000. Anais. Natal, Nov. 2000. CD ROM

SOUZA, B.I. Contribuição ao estudo da desertificação na bacia do rio Taperoá - PB. João Pessoa, Universidade Federal da Paraíba, 1999. 120p. (Tese de Mestrado)

van WIJK, ed. Physics of plant environment. Amsterdam, North Holland, 1963. 942p. 